\title{
Two new mutations in the dihydropteridine reductase gene in patients with tetrahydrobiopterin deficiency
}

\author{
Irma Dianzani, David W Howells, Alberto Ponzone, Jennifer A Saleeba, \\ Peter M Smooker, Richard G H Cotton
}

\begin{abstract}
Two new mutations have been identified within the dihydropteridine reductase (DHPR) gene in two patients with DHPR deficiency. The total coding sequence of the cDNA has been screened by chemical cleavage of mismatch in both patients and selected portions of the cDNA have been sequenced. The first mutation identified causes a glycine to aspartic acid substitution at codon 23 and seems particularly frequent in Mediterranean patients. Its occurrence within a glycine string common to the amino-terminal region in NADH dependent enzymes suggests a possible causal mechanism for the defect. The second change involves a tryptophan to glycine substitution at codon 108 and is carried by both alleles in the second patient. It occurs in a motif which shows similarities with a region of dihydrofolate reductase (DHFR) and is highly conserved within different animal species.
\end{abstract}

(f Med Genet 1993;30:465-9)

Dihydropteridine reductase (DHPR, EC 1.6.99.7) is an essential enzyme in the hydroxylating system of the aromatic amino acids, since it catalyses the regeneration of tetrahydrobiopterin $\left(\mathrm{BH}_{4}\right)$, the natural cofactor of phenylalanine, tyrosine, and tryptophan hydroxylases, from the quininoid-dihydrobiopterin produced in these coupled reactions. ${ }^{1-3}$

Inherited DHPR deficiency in humans causes hyperphenylalaninaemia as well as impaired production of monoamine neurotransmitters derived from tyrosine and tryptophan, dopamine, norepinephrine, and serotonin. ${ }^{4-7}$ This metabolic derangement leads patients to develop progressive and irreversible brain deterioration with severe neurological symptoms including psychomotor retardation, hypotonia of the trunk with limb hypertonia, and myoclonic epilepsy. A similar phenotype is shared by two additional inborn errors of $\mathrm{BH}_{4}$ synthesis, 6-pyruvoyl tetrahydropterin synthase and GTP cyclohydrolase deficiencies, responsible for impaired cofactor availability. On the basis of worldwide series, an overall frequency of such cases of 1 to $3 \%$ has been estimated among all types of hyperphenylalaninaemia detected by newborn mass screening. ${ }^{8}$ However, higher incidences have been reported in Mediterranean and non-Caucasian populations. ${ }^{9}$

All these disorders, at present called $\mathrm{BH}_{4}$ deficiency, are clinically unresponsive to a restricted phenylalanine diet, unlike phenylketonuria, and so they have been previously described as 'malignant' or 'atypical' hyperphenylalaninaemia. ${ }^{610}$ The unfavourable outcome can be largely prevented by the early administration of synthetic $\mathrm{BH}_{4}$, alone or in combination with diet and substitutive neurotransmitter therapy with hydroxylated precursors. ${ }^{11}$ The choice of the best treatment, and the individual adjustments required to obtain a good clinical response, are difficult to determine. Some DHPR deficient patients respond fully to $\mathrm{BH}_{4}$ administration, thus avoiding both low phenylalanine diets and neurotransmitter therapy, whereas others need neurotransmitter precursor supplementation. ${ }^{12}$ The clinical heterogeneity of DHPR deficiency has been confirmed by enzyme studies, leading to the identification of patients carrying a mutated protein devoid of catalytic activity $\left(\mathrm{DHPR}^{-} \mathrm{CRM}^{+}\right)$, and of others lacking any enzyme protein (DHPR ${ }^{-} \mathrm{CRM}^{-}$), or with different tissue distribution of residual activity. ${ }^{13-15}$

So far, our understanding of how DHPR functions is limited. Protein chemistry has shown that the native NADH dependent enzyme exists as a homodimer with a subunit molecular weight of between 25 and $27 \mathrm{kDa}^{16}$ Four cysteine residues are found in each subunit of the human and rat enzyme. ${ }^{17-20}$ The role of these cysteines is unclear; however, studies with bovine DHPR suggest that these residues do not form disulphide bridges. ${ }^{21}$ An alternate role for at least one sulphydryl group might be found in NADH binding since preincubation with NADH protects the enzyme against inhibition by a wide range of thiol modifying agents. ${ }^{161722}$

Further information and possible genotypephenotype correlations may arise from the identification of the underlying causal genetic lesions in patients with different clinical presentations.

The cDNA sequence (and by inference the amino acid sequence) of both human and rat DHPR have been determined ${ }^{18-20}$ and the rat DHPR has been shown to have a region of similarity with other NADH dependent enzymes. ${ }^{23}$

So far it seems that most cases of DHPR deficiency result from point mutations, since analysis of the restriction map excludes any large deletion occurring within the gene. Molecular heterogeneity is, however, expected for this disease, as inferred from the clinical presentation and Northern analysis. ${ }^{18}$ 
The identification of the molecular lesions responsible may be of great clinical value: identification of an association between phenotype and genotype may assist in a correct choice of therapy from birth. Furthermore, the identification of the molecular basis of DHPR deficiency may help to define the structure and organisation of the enzyme better. Finally, mutation detection may allow earlier prenatal diagnosis, now feasible only in the second trimester of pregnancy by evaluation of the enzyme activity in fetal erythrocytes.

Recently, the first causal mutation has been identified in a Turkish patient, the insertion of the triplet ACT (threonine) after alanine 122. ${ }^{24}$

We report here the characterisation of two new mutations within the DHPR gene in two patients with DHPR deficiency.

\section{Materials and methods \\ PATIENTS}

DHPR deficiency was diagnosed by measurement of urinary pterins and dried blood spot assay of DHPR in two patients. Case 1 was born to consanguineous parents of Portuguese origin. ${ }^{25}$ Hyperphenylalaninaemia was detected on neonatal screening and DHPR deficiency was diagnosed at 3 months of age. Cultured fibroblasts were $\mathrm{DHPR}^{-} \mathrm{CRM}^{+}$. Case 2 was diagnosed as having DHPR deficiency in the first month of life. ${ }^{9} \mathrm{He}$ was born to a mother from southern Italy who had a heterozygous level of DHPR activity. However, the father showed normal DHPR activity. Paternity testing was not performed.

\section{MUTATION DETECTION}

Mutation detection was achieved by the chemical cleavage of mismatch (CCM) method..$^{246}$ The DNA change was subsequently characterised by $\mathrm{M} 13$ subcloning ${ }^{27} 28$ and dideoxy chain termination sequencing. ${ }^{29}$

Total or messenger RNA from each patient was isolated from approximately $4 \times 10^{7}$ cul- tured skin fibroblasts, using a guaninidinium/ phenol/chloroform extraction technique, preceded by oligo(dT) cellulose to obtain poly(A)+RNA. RNA was resuspended in $\mathrm{H}_{2} \mathrm{O}$.

First strand cDNA was synthesised from $1 \mu \mathrm{g}$ of poly(A) + RNA or $20 \mu \mathrm{g}$ of total RNA using a cDNA synthesis kit (Amersham International plc). RNA secondary structure was minimised by heating to $65^{\circ} \mathrm{C}$ before cDNA synthesis.

The coding portion of the cDNA was either amplified in two overlapping sections using the primer pairs $A-D$ and $I-F$ or in one piece using the primers R114 and R113 (fig 1). Amplified cDNA from the patients was then mixed with similarly amplified and radiolabelled control DNA to generate a heteroduplex. Mismatch sites were cleaved using the chemical cleavage method. DHPR cDNA from two normal subjects was used as controls (KD4, S116); a further control corresponded to the human DHPR cDNA clone described by Dahl et al. ${ }^{18}$

For dideoxy chain termination sequencing the PCR products were purified by electrophoresis on a $3 \%$ Nusieve agarose gel and then subcloned into M13. Sequencing analysis was performed on several clones for each patient by means of the Sequenase sequencing kit (United States Biochemical Corporation).

\section{Results}

Chemical cleavage of a heteroduplex of case 2 and an internally labelled control probe derived from a human DHPR clone $^{18}$ (PCR products $\mathrm{R} 114-\mathrm{R} 113$, corresponding to the full length coding sequence) showed three cytosines that reacted with hydroxylamine. The first at approximately 90 bases from the end of the probe (fig $2 \mathrm{~A}$ ) corresponded to a $\mathrm{G}$ to $\mathrm{A}$ conversion at base 92 (fig 2B). This results in the change of the codon for Gly 23 (GGT) to an aspartic acid codon (GAT). The second faint band below the 92 bp mismatch (fig 2A)

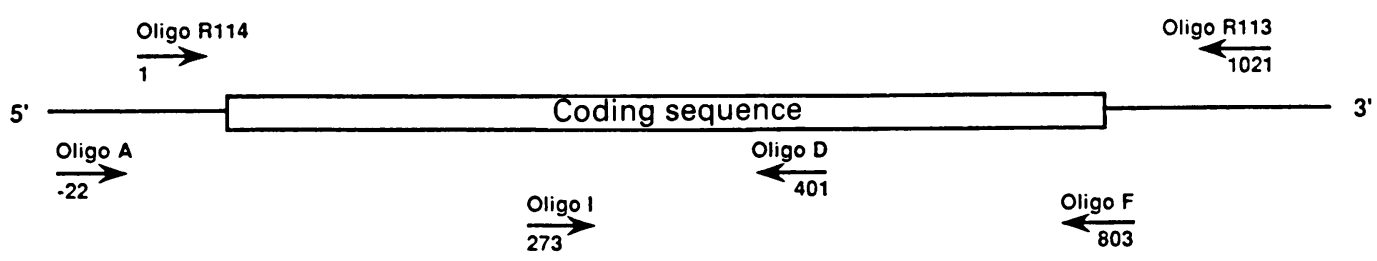

\begin{tabular}{|c|c|c|}
\hline Oligo A & 5 & ATAAGGGTTCGATTCGGAGC \\
\hline Oligo D & & GTAGACCGATGGTTCGTAGA 5 \\
\hline Oligo 1 & 5 & TCTTTGCGTTGCTGGAGGAT \\
\hline Oligo $\mathrm{F}$ & & CGGACGGTCTITTCAGTGAT 5 \\
\hline Oligo R114 & 5 & CGGAGCCGGGCTGGCAGGAG \\
\hline Oligo R113 & & TCCCGAGACGTATCGTGGAC \\
\hline
\end{tabular}

Figure 1 Diagrammatic representation of the coding sequence of the human DHPR cDNA showing pairs of oligonucleotide primers used for PCR amplification. The numbering (according to Dahl et al ${ }^{18}$ ) indicates the base in the cDNA which is complementary to the $5^{\prime}$ residue of the oligonucleotide. PCR was performed using the Cetus Perkin Elmer PCR protocol. For the primers $A-D$ and $I-F$ the final $M g C l$ concentrations were $2.5 \mathrm{mmol} / \mathrm{l}$ and $1.25 \mathrm{mmol} / \mathrm{l}$, respectively. Thirty five cycles of denaturation (two minutes at $95^{\circ} \mathrm{C}$ ), annealing (two minutes at $65^{\circ} \mathrm{C}$ ), and extension (three minutes at $72^{\circ} \mathrm{C}$ ) were performed. For the primers $R 114$ and $R 113,35 \mathrm{cycles}$ of denaturation (two minutes at $96^{\circ} \mathrm{C}$ ), annealing (two minutes, 30 seconds at $65^{\circ} \mathrm{C}$ ), and extension (three minutes at $72^{\circ} \mathrm{C}$ ) were performed at a final $\mathrm{MgCl}_{2}$ concentration of $1.25 \mathrm{mmol} / \mathrm{l}$. 
A

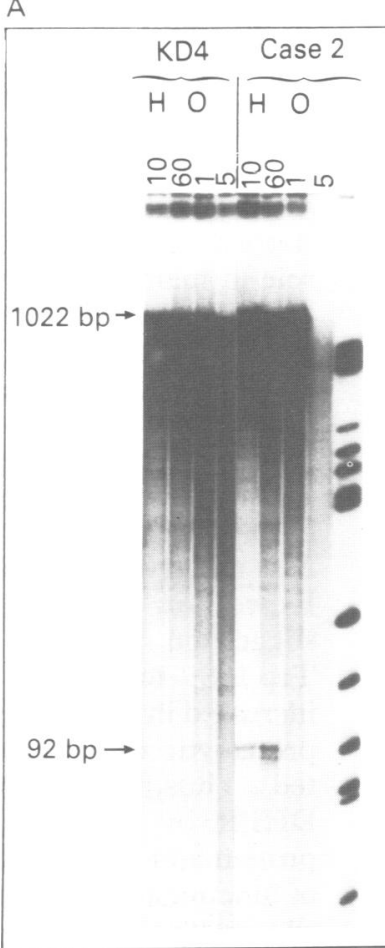

Figure 2 (A) CCM analysis of the entire coding sequence of DHPR (PCR primers R113 to R114) from the normal control KD4 and from case 2 showing hydroxylamine reactivity at $92 \mathrm{bp}$. The second faint band below corresponds to a secondary reaction from matched cytosine nearby. ${ }^{30}$ The probe, which was derived from the cDNA clone described by Dahl et al, ${ }^{18}$ was internally labelled by $P C R .{ }^{31} H$ and $O$ indicate hydroxylamine and osmium tetroxide reactions which were performed for 10 and 60 minutes and for one and five minutes respectively. (B) Sequence analysis between bases 79 and 102 for KD4 and case 2 showing the $G$ to $A$ conversion that changes the codon for Gly 23 to Asp 23.

corresponds to a secondary reaction from a matched cytosine nearby. ${ }^{30}$ The third reactive cytosine at approximately 490 bases from the end of the probe (results not shown) corresponds to a mutation at codon 158 and was also seen in another Italian DHPR deficient patient (Smooker et al, submitted). Expression studies showed this mutation to be causal (Smooker et al, submitted).

When end labelled PCR product I-F from the normal control KD4 was used as the probe to prepare heteroduplexes with case 1 and with a control S116, both showed reactivity with hydroxylamine indicating a mismatched cytosine approximately $126 \mathrm{bp}$ from the end of the probe (fig 3A). Sequencing of this region showed a $G$ to $A$ conversion at base 420 changing CTG to CTA, conserving the Leu 132 codon (fig 3C). No other CCM reactivity was seen for case 1 with either hydroxylamine or osmium tetroxide in IF or AD PCR products. However, sequencing of the I-F PCR product of case 1 showed a $T$ to $G$ conversion at base 346 that changed the codon for tryptophan 108 (TGG) to a glycine codon (GGG) (fig 3B). This $T$ to $G$ alteration (which forms a $T / C$ mismatch with the $T$ in the labelled strand) was not detected by the CCM method, probably because of the high stability of the surrounding G/C base pairs (Cotton et $a l$, submitted). This problem can be overcome by using a slightly different CCM proced-

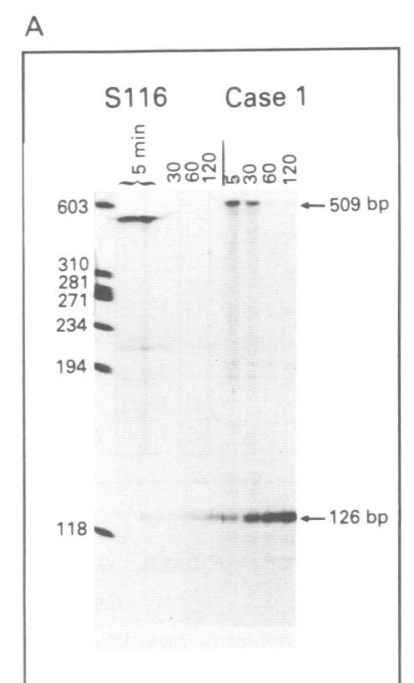

B

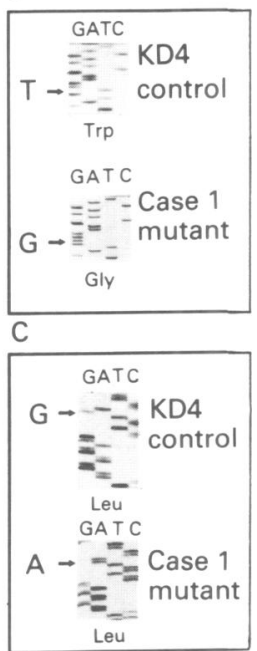

Figure 3 (A) CCM gel showing hydroxylamine reactivity approximately $126 \mathrm{bp}$ from the end of the probe in the I-F PCR products of the normal control $S 116$ and case 1 . The probe was obtained from similarly amplified KD4 cDNA. Restriction digestion of the control DNA with Sau961 gave a staggered cut that was filled in with ${ }^{32} P d C T P$ using the Klenow fragment of DNA polymerase I to give an end labelled probe. Reactions were performed for five, 30,60, and 120 minutes. The fainter reactivity showed by control S116 is because of heterozygosity for the corresponding DNA change. ${ }^{24}(B)$ Sequence analysis of the I-F PCR product of case 1 and the normal control KD4 between bases 341 and 359 showing the $T$ to $G$ conversion at base 346 that changes the codon for Trp 108 (TGG) to a glycine codon (GGG). (C) Sequence analysis between bases 399 and 426 from the DHPR deficient case 1 and from the normal control KD4 (used as probe for the $C C M$ reactions in $(A))$ showing the $T$ to $A$ conversion at base $\mathbf{4 2 0}$ which corresponds to the hydroxylamine reactivity. This conversion changes the normal leucine codon (CTG) to an alternative leucine codon (CTA).

ure. ${ }^{3233}$ Since all the five sequenced M13 clones carried this change at codon 108 and since case 1 was born to consanguineous parents, he is homozygous for this mutation.

\section{Discussion}

In this paper we report two new mutations affecting the DHPR gene in two patients with DHPR deficiency.

The mutation in case 2 is a conversion of glycine to aspartate at codon 23 . This glycine is located within a highly conserved 'glycine fingerprint' found in human DHPR (fig 4) and common to most NADH binding enzymes at their amino-terminal. ${ }^{34}{ }^{35}$ In these enzymes a $\beta-\alpha-\beta$ fold is centred around a highly conserved sequence Gly-X-Gly-X-X-Gly (where $X$ is any amino acid) that constitutes a tight turn at the end of the first strand of a $\beta$ sheet and marks the beginning of the subsequent $\alpha$ helix. The first glycine residue is essential for the tightness of the turn, the second allows the dinucleotide to be bound without obstruction from an amino acid side chain at this position, and the third provides space to allow close association between the $\beta$ strands and the $\alpha$ helix. ${ }^{35}$ Glycine at codon 23 in DHPR corresponds to the third of these glycines. Thus it may be expected that the insertion of the bulkier and negatively charged aspartic acid disrupts the geometry of a possible NADH binding site and suggests a mechanism for DHPR deficiency in case 2 . It is interesting in 


\begin{tabular}{lll}
\hline Protein $\quad$ Organism & $\begin{array}{l}\text { First } \\
\text { residue }\end{array}$ & Sequence \\
\hline
\end{tabular}

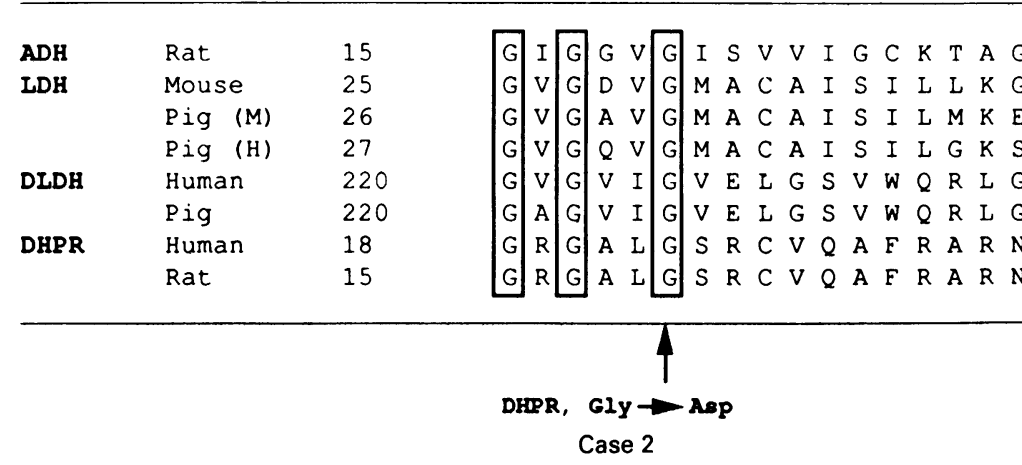

Figure 4 Comparison of the sequences of human and rat DHPR with the glycine fingerprint region implicated in NADH binding from a variety of enzymes. The arrow indicates the mutation site in case $2 . A D H=$ aldehyde dehydrogenase, $L D H=$ lactate dehydrogenase, $D L D H=$ dihydrolipoamide dehydrogenase.

this regard to note that a role in NADH binding has been invoked for one of the four cysteines included in DHPR, since preincubation with NADH appeared to protect the enzyme against inhibition by thiol modifying agents. The cysteine at codon 26 might be the residue involved in this process.

Further studies are needed to confirm this hypothesis. However, enzymology shows that the Gly 23 Asp mutant protein expressed in Escherichia coli has no DHPR activity (Smooker et al, submitted).

Case 2 responds so well to $\mathrm{BH}_{4}$ monotherapy that he is now on a free phenylalanine intake. ${ }^{12}$ This might be because of the presence of residual activity of the mutated enzyme which could be forced by a high concentration of substrate. Further studies are needed to elucidate the relevance of each of the mutations in case 2 to this effect.

Codon 108 lies in a sequence bearing similarities to a small region of DHFR, ${ }^{18}$ which contains six amino acids that are invariant in DHFR from a wide range of vertebrates suggesting functional importance ${ }^{36}$ (fig 5). Mutation of one of these conserved residues, Trp 108 to glycine, was identified in case 1 and, interestingly, the threonine insertion immediately after Ala 122, the first mutation recognised in a DHPR deficient gene, also lies in this region ${ }^{24}$ (fig 5).

In DHFR substitution of phenylalanine for Trp 24, corresponding to Trp 108 in DHPR, results in a more flexible conformation that shows general reduction in affinity for its substrates, ${ }^{37}$ suggesting that this residue plays a role in maintaining the structural integrity of DHFR. On the other hand, in rat DHPR substitution of phenylalanine for $\operatorname{Trp} 104$ (the residue equivalent to human DHPR, Trp 108) increased the $\mathrm{Km}$ for NADH but did not significantly reduce Kcat. However, it is important to observe that in both these cases the mutation is conservative, since both phenylalanine and tryptophan are aromatic amino acids. Expression studies fit well with the former suggestion, since the DHPR protein with Trp 108 to Gly substitution shows an increased in vitro susceptibility to digestion by proteolytic enzymes (Smooker et al, submitted). Thus, this mutation probably inactivates DHPR by causing aberrant folding of the protein and consequent disruption of a region of functional importance. The hypothesis of an encoded non-functional protein is in agreement with the finding of CRM + material within fibroblasts from this patient, homozygous for the Trp to Gly substitution.

Finally, our study identified the change of the CTG codon for Leu 132 to CTA, an alternate leucine codon, in case 1 and in control S116 (fig 2). This change has been reported previously in another child with DHPR deficiency indicating that this is a common polymorphism. ${ }^{24}$ However, even harmless polymorphisms may be of clinical value in populations (or for diseases) where most of the causal mutations are unknown, since they allow the mutant allele to be traced in family studies.

In conclusion, two new mutations have been characterised in two patients with DHPR deficiency. The localisation of the mutations within highly conserved amino acid motifs within the protein and the preliminary results of expression studies suggest that they are most probably causal. Identification of the Gly 23 to Asp substitution in several patients of Mediterranean origin suggests that this mutation may be the most frequent molecular

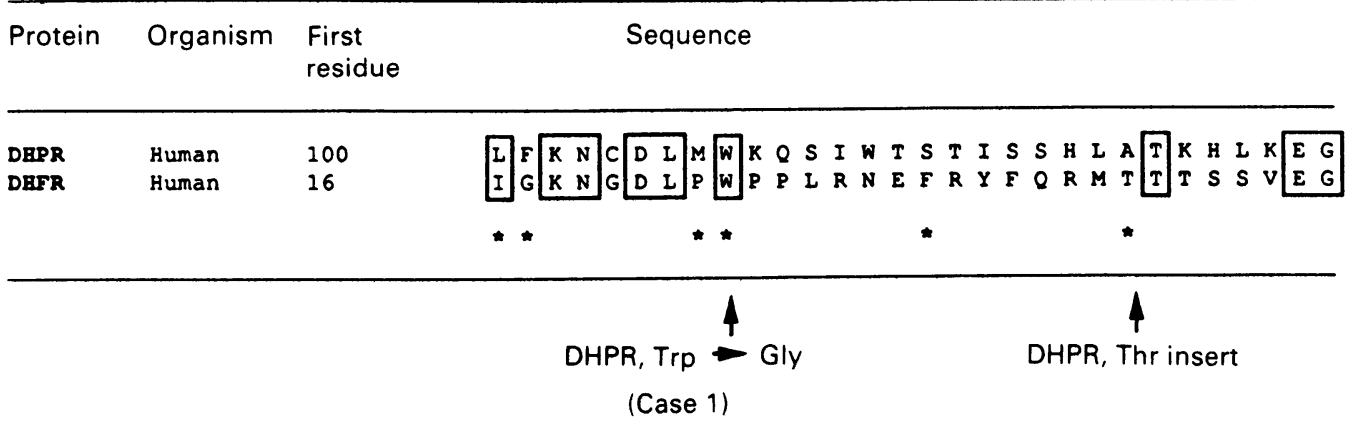

Figure 5 Comparison of the sequences of DHPR and DHFR in a highly conserved portion of DHFR. Boxes indicate the conserved residues, asterisks indicate residues absolutely conserved in several species of DHFR. ${ }^{18}$ The arrows indicate the sites of mutations causing DHPR deficiency in case 1 and in the patient described by Howells et $a l .{ }^{24}$ 
cause of DHPR deficiency in Mediterranean populations (Smooker et al, submitted).

We thank Dr Clara Camaschella for criticising the manuscript and for helpful discussion and Dr Françoise Rey for supplying the fibroblasts from case 1 . This work was partially supported by the National Australian Medical Research Foundation.

1 Craine JE, Hall ES, Kaufman S. The isolation and characterization of dihydropteridine reductase from sheep liver. f Biol Chem 1972;247:6082-91.

2 Benkovic SJ. On the mechanism of action of folate- and biopterin-requiring enzymes. Annu Rev Biochem 1980; 49:227-51.

3 Lazarus RA, Benkovic SJ, Kaufman S. Phenylalanine hydroxylase stimulator protein in a 4-carbinolamine dehydratase. F Biol Chem 1983;258:10960-2.

4 Koslow SH, Butler IJ. Biogenic amine synthesis defect in dihydropteridine reductase deficiency. Science 1977; 198:522-3.

5 Smith I. Atypical phenylketonuria with progressive neurological illness unresponsive to dietary treatment. Arch Dis Child 1974;49:245-8.

6 Blau N. Inborn errors of pterin metabolism. Annu Rev Nutr 1988;8:185-209.

7 Smith I, Howells DW, Hyland K. Pteridines and monoamines: relevance to neurological damage. Postgrad Med $\mathcal{F}$ 1986;62:113-23.

8 Dhont JL. Tetrahydrobiopterin deficiency: preliminary analysis from international survey. $\mathcal{f}$ Pediatr 1984; 104:501-8.

9 Ponzone A, Guardamagna O, Ferraris S, Ferrero GB, Dianzani I, Cotton RGH. Tetrahydrobiopterin loading test in hyperphenylalaninemia. Pediatr Res 1991;30:4358.

10 Danks DM, Bartholome K, Clayton RE, et al. Malignant hyperphenylalaninemia, current status. F Inher Metab Dis 1978;1:49-53.

11 Ponzone A, Ferrero GB, Guardamagna O, Ferraris S, Curtius HC, Blau N. Screening and treatment of tetrahydrobiopterin deficiency. In: Curtius HC, Ghisla S, Blau N, eds. Chemistry and biology of pteridines. Berlin: Walter de Gruyter, 1990:393-4.

12 Ponzone A, Guardamagna O, Spada M, Ferrero GB, Dianzani I, Cotton RGH. Catalytic activity of tetrahydrobiopterin in dihydropteridine reductase deficiency and indications for treatment. Pediatr Res 1993;33:125-8.

13 Nakabayashi H, Owada M, Kitagawa T. A mild case of dihydropteridine reductase deficiency with residual activity in erythrocytes. F Inher Metab Dis 1984;7:135-6.

14 Cotton RGH, Jennings I, Bracco G, Ponzone A, Guardamagna $O$. Tetrahydrobiopterin non-responsiveness in dihydropteridine reductase deficiency is associated with presence of mutant protein. $\mathcal{F}$ Inher Metab Dis 1986; 9:239-43.

15 Ponzone A, Guardamagna O, Ferraris S, Bracco G, Niederwieser A, Cotton RGH. Two mutations of dihydropteridine reductase deficiency, Arch Dis Child 1987;63:154-7.

16 Firgaira FA, Cotton RGH, Danks DM. Isolation and characterization of dihydropteridine reductase from human liver. Biochem $\mathcal{f} 1981 ; 197: 31-43$.

17 Webber S, Whiteley JM. The effect of specific amino acid modifications on the catalytic properties of rat liver modifications on the catalytic properties of rat liver
dihydropteridine reductase. Arch Biochem Biophys 1981; 206:145-52.

18 Dahl HHM, Hutchinson W, McAdam W, Wake S, Morgan FJ, Cotton RGH. Human dihydropteridine reductase: characterisation of a cDNA clone and its use in analysis of patients with dihydropteridine reductase deficiency. Nucpatients with dihydropteridine
leic Acids Res 1987;15:1921-32.

19 Lockyer J, Cook RG, Milstien S, Kaufman S, Woo SLC Ledley FD. Structure and expression of human dihydropteridine reductase. Proc Natl Acad Sci USA 1987;84:3329-33.

20 Shabbaz M, Hoch JA, Trach KA, Hural JA, Webber S, Whitely JM. Structural studies and isolation of cDNA clones providing the complete sequence of rat liver dihydropteridine reductase. $\mathcal{F}$ Biol Chem 1987;262:16412-16.

21 Aksnes A, Skotland T, Flatmark T, Ljones T. Bovine dihydropteridine reductase purification by affinity chromatography and comparison of enzymes from liver and adrenal medulla. Neurochem Res 1979;4:385-98.

22 Cheema S, Soldin SJ, Knapp A, Hofman T, Scrimgeour KG. Properties of purified quininoid dihydropterin reductase. Can 7 Biochem 1973;51:1229-39.

23 Matthews DA, Varughese KI, Skinner M, et al. Role of aspartate-37 in determining cofactor specificity and binding in rat liver dihydropteridine reductase. Arch Biochem ing in rat liver dihydropter.

24 Howells DW, Forrest SM, Dahl HHM, Cotton RGH Insertion of an extra codon for threonine is a cause of dihydropteridine reductase deficiency. Am $\mathcal{F}$ Hum Gene 1990;47:279-85.

25 Rey F, Harpey JP, Leeming RJ, Blair JA, Aicardi J, Rey J. Les hyperphenylalaninemies avec activité normale de la phenylalanine hydroxylase. Le deficit en tetrahydrobiopterine et le deficit en dihydropteridine reductase. Arch Fr Pediatr 1977;34: CIX-CIXX

26 Cotton RGH, Rodrigues NR, Campbell RD. Reactivity of cytosine and thymine in single-base-pair mismatches with hydroxylamine and osmium tetroxide and its application to the study of mutations. Proc Natl Acad Sci USA to the study of mutt
$1988 ; 85: 4397-401$.

27 Messing J. New M13 vectors for cloning. Methods Enzymol 1983;101:20-78.

28 Norrander J, Kempe T, Messing J. Construction of improved $M 13$ vectors using oligodeoxynucleotide-directed mutagenesis. Gene 1983;26:101-6.

29 Sanger F, Nicklen S, Coulson AR. DNA sequencing with chain-terminating inhibitors. Proc Natl Acad Sci USA 1977;74:5463-7.

30 Cotton RGH, Campbell RD. Chemical reactivity of matched cytosine and thymine bases near mismatched and unmatched bases in a heteroduplex between DNA strands with multiple differences. Nucleic Acids Res 1989;17:4223-33.

31 Dianzani I, Camaschella C, Saglio G, Forrest SM, Ramus $S$, Cotton RGH. Simultaneous screening for $\beta$ thalassemia mutations by chemical cleavage of mismatch. Genomics 1991;11:48-53.

32 Forrest SM, Dahl HHM, Howells DW, Dianzani I, Cotton RGH. Mutation detection in phenylketonuria using the chemical cleavage method: importance of using probes from both normal and patient samples. Am $\mathcal{F}$ Hum Genet 1991;49:175-83.

33 Saleeba J, Ramus S, Cotton RGH. Complete mutation detection using unlabeled chemical cleavage. Hum Mutat 1992;1:63-9.

34 Wierenga RK, DeMaeyer MCH, Hol WGJ. Interaction of pyrophosphate moieties with alpha-elixes in dinucleotide binding proteins. Biochemistry 1985;24:1346-57.

35 Scrutton NS, Berry A, Perham RN. Redesign of the coenzyme specificity of a dehydrogenase by protein engineering. Nature 1990;343:38-43.

36 Bzik DJ, Li WB, Horii T, Inselburg J. Molecular cloning and sequence analysis of the Plasmodium falciparum and sequence analysis of the Plasmodium falciparum Natl Acad Sci USA 1987;84:8360-4.

37 Huang SM, Delcamp TJ, Tan XH, Smith PL, Prendergast NJ, Freisheim JH. Effects of conversion of an invariant tryptophan residue to phenylalanine on the function of human dihydrofolate reductase. Biochemistry 1989 28:471-8. 